\title{
REDUCCIÓN DE LA FERTILIZACIÓN INORGÁNICA MEDIANTE MICORRIZA ARBUSCULAR EN SORGO
}

Reduction of inorganic fertilization through arbuscular mycorrhiza in sorghum

\author{
Arturo DÍAZ FRANCO, Martín ESPINOSA RAMÍREZ y Flor Elena ORTIZ CHÁIREZ*
}

Instituto Nacional de Investigaciones Forestales, Agrícolas y Pecuarias, Campo Experimental Río Bravo, km 61 carretera Matamoros-Reynosa, Apdo. Postal 172, Ciudad Río Bravo, 88900 Río Bravo, Tamaulipas, México *Autora para correspondencia: ortiz.flor@inifap.gob.mx

(Recibido enero 2018; aceptado septiembre 2018)

Palabras clave: Sorghum bicolor, características de planta, productividad

\section{RESUMEN}

Dentro de la conservación y la fertilidad de los suelos se ha destacado el papel de los hongos micorrízicos arbusculares (HMA). El objetivo del presente estudio fue conocer la respuesta de los sorgos DKS60 en 2011 y $83 \mathrm{G} 19$ en 2016, en cuanto a características fisiológicas y productivas, inoculado con HMA (Rhizophagus intraradices) solo o en combinación con la mitad de la fertilización inorgánica (FI50 \%), comparada con la fertilización convencional (120N-40P-00K; FI100 \%). Con el rendimiento del grano se estimó la rentabilidad de la producción mediante el indicador beneficio-costo (b/c). Todos los tratamientos superaron significativamente al testigo respecto de índice de clorofila (SPAD), altura de planta, diámetro de tallo, longitud de panoja y rendimiento del grano, aunque en general los mayores valores se registraron con FI100 \% y FI50 \% + HMA. No obstante, en el b/c se demostró que FI50 \% + HMA y HMA sólo obtuvieron 1.45 y $1.5 \mathrm{~b} / \mathrm{c}$, respectivamente, superiores a la rentabilidad obtenida con FI100 \% (1.3). Lo anterior, a pesar de que el HMA registró $852 \mathrm{~kg} /$ ha menos que el promedio de FI100 $\%$ y FI50 \% + HMA. La reducción o sustitución de la fertilización inorgánica a través de HMA puede representar una práctica viable que promueva mayor rentabilidad, así como la conservación agroecológica de los sistemas de producción.

Key words: Sorghum bicolor, plant characteristics, productivity

\begin{abstract}
The role of arbuscular mycorrhizal fungi (AMF) has been emphasized in the fields of conservation and soil fertility. The objective of this study was to explore the response of sorghum DKS60 in 2011 and 83G19 in 2016 - with respect to plant physiology and productivity - to AMF (Rhizophagus intraradices) alone or combined with half of inorganic fertilizer (IF50 \%), compared with conventional fertilization (120N-40P00K; IF100 \%). Profitability was estimated through grain yield with a benefit-cost (b/c) indicator. All treatments exceeded significantly the control with respect to chlorophyll index (SPAD), plant height, stem diameter, panicle length and grain yield, although overall the highest values were recorded with IF100 \% and IF50\%+ AMF. However, the benefit-cost relationship demonstrated that IF50 \% + HMA and AMF alone obtained
\end{abstract}


1.45 and $1.5 \mathrm{~b} / \mathrm{c}$, respectively, higher than the profitability obtained with IF-100 \% (1.3). This, although AMF alone recorded $852 \mathrm{~kg} /$ ha less than the average of IF $100 \%$ and IF50 \% + AMF. Reduction or replacement of inorganic fertilization through AMF may represent a viable practice promoting greater profitability, as well as the conservation of agro-ecological production systems.

\section{INTRODUCCIÓN}

En México, el estado de Tamaulipas se ubica como el principal productor de sorgo para grano (Sorghum bicolor) con participación de $40 \%$ y un volumen de dos millones de toneladas. La mayor superficie se siembra en la región norte del estado con 650000 ha anuales. En la mayoría de los casos se siembra en monocultivo, y alrededor del $30 \%$ en condiciones de riego (FIRA 2016). Las limitaciones nutrimentales del cultivo se cubren mediante la fertilización mineral. Rosales et al. (2006) sugieren para el sorgo en condiciones de riego la adición de 120 y $40 \mathrm{~kg} / \mathrm{ha}$ de N y P, respectivamente. Por otro lado, el alto costo de la práctica de fertilización induce a la vez incrementos en los costos de la producción; además, su uso continuo ha tenido como resultado contaminación, decremento de la biodiversidad en las regiones agrícolas y degradación de los agroecosistemas (Grageda et al. 2012, Xiang et al. 2012). Se estima que entre 10 y $40 \%$ de los fertilizantes aplicados son utilizados por las plantas y el resto constituyen contaminantes en el proceso de lixiviación, además de las emisiones de dióxido de carbono y óxidos de nitrógeno a la atmósfera (Adesemoye y Kloepper 2009). Ante este contexto, se ha resaltado la demanda de desarrollar prácticas agronómicas que eleven la productividad del sorgo, pero que además promuevan un equilibrio amigable con el ambiente (Williams 2006, Díaz et al. 2007, Díaz et al. 2013).

Una posible solución a este problema agroambiental son los sistemas de gestión integrada de los nutrientes que complementan la fertilización con biofertilizantes. Por ello, en respuesta a la necesidad de un manejo sostenible de los sistemas agrícolas, se ha incrementado de forma prominente el papel de los microorganismos a manera de bioinoculantes para gestionar la conservación y fertilidad de los suelos (Sharma et al. 2012, Malusá et al. 2016).

Los hongos micorrízicos arbusculares (HMA) forman asociaciones de tipo mutualista con las plantas y contribuyen de manera importante al mantenimiento de la calidad de los suelos, ya que dentro de su actividad simbiótica manifiestan diferentes mecanismos que inducen a una mayor exploración del suelo a través de hifas. Asimismo, disminuyen los efectos de condiciones abióticas adversas para la planta, producen fitohormonas que estimulan el crecimiento de la planta, facilitan la absorción de nutrimentos y estimulan una acción protectora contra algunos fitopatógenos del suelo. Dicha asociación desempeña también un papel importante sobre las características físicas del suelo, al incrementar la agregación de partículas y estabilidad de éste (Smith y Read 2008, Yang et al. 2014). No obstante, el nivel de la colonización fúngica radical no siempre está asociado con la efectividad exhibida, esto es, con el rendimiento del cultivo o sus componentes (Sylvia et al. 1993, Allen et al. 2001, Díaz et al. 2008). En un estudio con parcelas comerciales de sorgo no fertilizadas, la inoculación del HMA Rhizophagus intraradices incrementó $47 \%$ la biomasa radical, $14 \%$ la longitud de panoja y $20 \%$ el rendimiento de grano, respecto a las plantas no inoculadas (Díaz et al. 2013).

El efecto benéfico de la micorrización podría tener además repercusiones ambientales y económicas favorables, ya que reduce o sustituye las necesidades de fertilizantes, por lo que es un tema necesario de estudio. Al respecto, Berruti et al. (2015), Rouphael et al. (2015) y Malusá et al. (2016) han investigado los efectos comparativos de bioinoculantes con la aplicación de fertilizantes minerales en los cultivos. Rose et al. (2014) indicaron que la biofertilización puede reducir las necesidades de nitrógeno hasta 52 $\%$ en arroz (Oryzae sativa). En un estudio previo con sorgo, Díaz et al. (2015) concluyeron que algunos bioinoculantes comerciales combinados con la mitad de la fertilización inorgánica, mostraron competitividad productiva respecto al manejo de fertilización convencional. Por lo anterior, el objetivo del estudio fue conocer la respuesta del sorgo a la inoculación de semilla con HMA combinada con la mitad de la fertilización inorgánica en condiciones de riego.

\section{MATERIALES Y MÉTODOS}

Los experimentos se realizaron durante los ciclos otoño-invierno de 2011 y 2016 en condiciones de rie- 
go, en los terrenos del Campo Experimental Río Bravo (CERIB) del Instituto Nacional de Investigaciones Forestales, Agrícolas y Pecuarias (INIFAP), ubicado

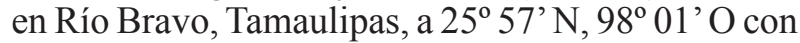
altitud de $25 \mathrm{msnm}$, clima semicálido, temperatura media anual de $23.5^{\circ} \mathrm{C}$ y $648 \mathrm{~mm}$ de precipitación (Silva et al. 2007). Antes de las siembras se hicieron muestreos de suelo y en el Laboratorio de Suelo y Planta del CERIB, se analizaron las propiedades físicas y químicas (SEMARNAT 2002), resultados contenidos en el cuadro I.

\section{Manejo experimental en 2011}

El híbrido de sorgo utilizado fue DKS60, sembrado el 1 de febrero de 2011 en humedad residual y a

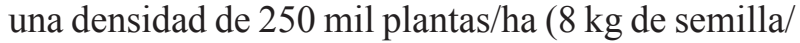
ha). Los tratamientos evaluados fueron: 1) fertilización química convencional, 120 y $40 \mathrm{~kg} / \mathrm{ha}$ de $\mathrm{N} \mathrm{y}$ P, respectivamente (FI100 \%) (Rosales et al. 2006); 2) la mitad de la fertilización inorgánica (FI50 \%) más semilla inoculada con el HMA Rhizophagus intraradices, micorriza INIFAP $(0.5 \mathrm{~kg} / \mathrm{ha}) ; 3)$ semilla inoculada con el HMA, y 4) testigo absoluto. Los tratamientos se distribuyeron en un diseño de bloques completos al azar con cuatro repeticiones. Las parcelas estaban conformadas por cuatro surcos $(0.81 \mathrm{~m})$ y $5 \mathrm{~m}$ de longitud. La fertilización con la mezcla de urea y superfosfato triple de calcio se incorporó lateralmente en el surco al momento de la siembra. La aplicación de $\mathrm{N}$ fue fraccionada, la mitad se agregó en la siembra y la otra mitad antes del primer riego de auxilio. Se aplicaron dos riegos de auxilio, a los 40 y 65 días de la emergencia de las plántulas. Otras prácticas agronómicas se efectuaron según indicaciones locales (Rosales et al. 2006).

En el estado de hoja bandera de las plantas, se estimó el índice de clorofila (SPAD) en los surcos centrales de las parcelas mediante el promedio de 10 lecturas tomadas en la parte central de la hoja bandera con un medidor portátil Minolta SPAD-502 ${ }^{\circledR}$. En ese mismo estado se evaluó la altura de cinco plantas por parcela. En madurez fisiológica, y con el mismo número de plantas, se midió la altura de la planta, el diámetro del tallo y la longitud de la panoja. En la cosecha, el rendimiento se obtuvo del total de panojas de los dos surcos centrales por parcela; las panojas se secaron bajo el sol y se trillaron. El rendimiento de grano se ajustó al $12 \%$ de humedad. Los efectos de los tratamientos sobre las variables se determinaron por análisis de varianza y para la comparación de promedios entre tratamientos se utilizó la prueba de Tukey $(\mathrm{p} \leq 0.05)$ mediante el programa SAS (v. 8.1).

\section{Manejo experimental en 2016}

El híbrido de sorgo utilizado fue $83 \mathrm{G} 19$, sembrado el 15 de febrero de 2016 ( $8 \mathrm{~kg}$ de semilla/ha), para lo cual se requirió un riego de presiembra. Los tratamientos evaluados fueron los mismos que en 2011. Los tratamientos se distribuyeron en un diseño de bloques completos al azar con tres repeticiones. Las parcelas se conformaron por tres surcos $(0.81$ $\mathrm{m})$ y $5 \mathrm{~m}$ de longitud. El fertilizante a base de urea y fosfato diamónico (DAP) se adicionó a un lado del surco en la siembra. El $\mathrm{N}$ fue fraccionado, la mitad en la siembra y la otra parte en el primer riego de auxilio. Se aplicaron dos riegos de auxilio, a los 42 y 61 días de la emergencia de las plántulas. Otras prácticas agronómicas se siguieron conforme al paquete tecnológico de la región (Rosales et al. 2006).

Las variables de respuesta fueron SPAD, altura de planta, biomasa seca foliar y radical, diámetro de tallo, longitud de panoja y rendimiento de grano. El SPAD se obtuvo de 10 lecturas por parcela en los estados fenológicos de cinco hojas, hoja bandera y floración. Los registros de altura de planta fueron de cinco plantas por parcela en los estados de hoja bandera y madurez fisiológica. Los datos de las variables se obtuvieron de los surcos centrales de las parcelas. Asimismo, el rendimiento de grano se estimó a partir de las panojas del surco central, secadas al sol y trilladas. El grano se ajustó al $12 \%$ de humedad. Los datos se sometieron a análisis de varianza y para las diferencias entre las medias se utilizó la prueba de Tukey $(\mathrm{p} \leq 0.05)$ a través del programa SAS (v. 8.1).

CUADRO I. PROPIEDADES FÍSICAS Y QUÍMICAS DEL SUELO PREVIAS AL ESTABLECIMIENTO DE LOS EXPERIMENTOS

\begin{tabular}{lccccccc}
\hline Año & $\mathrm{pH}$ & $\begin{array}{c}\mathrm{CE} \\
(\mathrm{dS} / \mathrm{m})\end{array}$ & $\begin{array}{c}\mathrm{MO} \\
(\%)\end{array}$ & $\begin{array}{c}\mathrm{N} \\
(\mathrm{mg} / \mathrm{kg})\end{array}$ & $\begin{array}{c}\mathrm{P} \\
(\mathrm{mg} / \mathrm{kg})\end{array}$ & $\begin{array}{c}\mathrm{K} \\
(\mathrm{mg} / \mathrm{kg})\end{array}$ & Textura \\
\hline 2011 & 7.8 & 1.10 & 1.5 & 23.5 & 23.9 & 725 & Franco arcillosa \\
2016 & 7.9 & 1.14 & 1.7 & 20.6 & 29.0 & 1869 & Arcillosa \\
\hline
\end{tabular}

C.: conductividad eléctrica, MO: materia orgánica 


\section{Experimentos 2011 y 2016}

Con las variables coincidentes en los estados fenológicos de ambos experimentos (hoja bandera: SPAD y altura de planta; madurez fisiológica: altura de planta, diámetro de tallo, longitud de panoja y rendimiento de grano), se realizaron correlaciones de Pearson con las ecuaciones correspondientes. Adicionalmente, se calculó la rentabilidad de la producción actual empleando como indicador económico la relación beneficio-costo $(\mathrm{b} / \mathrm{c})$ (costo de producción $\$ 12$ $261 /$ ha; precio medio rural $\$ 2240 /$ t), la cual indica los beneficios obtenidos por cada peso invertido en la producción.

\section{RESULTADOS}

En 2011 los tratamientos no influyeron en el SPAD en el estado de hoja bandera del sorgo DKS60. Por el contrario, en sorgo 83G19 el SPAD mostró variaciones significativas en los tres estados de desarrollo de la planta y se observaron incrementos ascendentes en sus valores conforme al desarrollo de la misma. En estado de cinco hojas y en hoja bandera, los tres tratamientos evaluados superaron significativamente $(\mathrm{p}=0.03)$ al testigo; en floración, los mayores valores $(\mathrm{p}=0.02)$ se registraron con la fertilización convencional (FI100 \%) y FI50 \% más la inoculación micorrízica, aunque el HMA solo superó al testigo (Cuadro II).

La altura de planta en estado de hoja bandera en el experimento de 2011 (DKS60) fue semejante entre los tratamientos. En madurez fisiológica hubo diferencias significativas en la altura de planta $(p=0.01)$, el diámetro de tallo $(\mathrm{p}=0.04)$, la longitud de panoja $(p=0.04)$ y el rendimiento de grano $(p=0.001)$. En altura de la planta destacó el tratamiento con HMA y fue el único que superó al testigo. En el diámetro de tallo todos los tratamientos superaron al testigo, los cuales promediaron $16.1 \mathrm{~mm}, 2.43 \mathrm{~mm}$ más que el testigo. Para la longitud de panoja y rendimiento de grano, la combinación FI50 \% más el HMA registró los valores mayores (Cuadro III).

En el experimento de 2016, la altura de planta en etapa de hoja bandera, al igual que en el año anterior señalado, no fue influenciada por los tratamientos;

CUADRO II. ÍNDICE DE CLOROFILA (SPAD) EN 2011 Y 2016 INFLUENCIADO POR LA FERTILIZACIÓN INORGÁNICA (FI) Y/O INOCULACIÓN MICORRÍZICA (HMA)

\begin{tabular}{|c|c|c|c|c|}
\hline \multirow[t]{3}{*}{ Tratamiento } & \multicolumn{4}{|c|}{ Clorofila SPAD } \\
\hline & \multirow{2}{*}{$\begin{array}{l}\text { 2011/DKS60 } \\
\text { Hoja bandera }\end{array}$} & \multicolumn{3}{|c|}{ 2016/83G19 } \\
\hline & & Cinco hojas & Hoja bandera & Floración \\
\hline 120-40-00 (FI100 \%) & $43.4 \pm 2.7$ & $34.5 \pm 0.9 \mathrm{a}^{*}$ & $43.4 \pm 1.7 \mathrm{a}$ & $49.7 \pm 2.0 \mathrm{a}$ \\
\hline FI50 \% + HMA & $45.7 \pm 1.6$ & $34.2 \pm 1.1 \mathrm{a}$ & $43.5 \pm 4.0 \mathrm{a}$ & $48.5 \pm 2.0 \mathrm{a}$ \\
\hline HMA & $41.3 \pm 1.0$ & $34.2 \pm 1.2 \mathrm{a}$ & $41.0 \pm 1.3 \mathrm{~b}$ & $44.2 \pm 3.3 b$ \\
\hline Testigo & $40.8 \pm 0.9$ & $32.1 \pm 0.7 \mathrm{~b}$ & $36.6 \pm 1.5 \mathrm{c}$ & $39.9 \pm 4.0 \mathrm{c}$ \\
\hline Significancia $F$ & 0.11 & 0.03 & 0.03 & 0.02 \\
\hline
\end{tabular}

*Letras distintas denotan diferencias significativas con la prueba de Tukey $(\mathrm{p}<0.05)$

FI100 \% = fertilización inorgánica $100 \%$ (120-40-00), FI50 \% = fertilización inorgánica $50 \%$

CUADRO III. RESPUESTA DEL SORGO DKS60 (2011) EN ESTADO DE HOJA BANDERA Y MADUREZ FISIOLÓGICA CON DIFERENTE MANEJO DE FERTILIZACIÓN (FI) Y/O INOCULACIÓN DEL HMA

\begin{tabular}{lccccc}
\hline Tratamiento & \multicolumn{2}{c}{ Altura de planta $(\mathrm{cm})$} & \multirow{2}{*}{$\begin{array}{c}\text { Diámetro de tallo } \\
(\mathrm{cm})\end{array}$} & $\begin{array}{c}\text { Longitud de panoja } \\
(\mathrm{cm})\end{array}$ & $\begin{array}{c}\text { Rendimiento } \\
(\mathrm{kg} / \mathrm{ha})\end{array}$ \\
\cline { 2 - 3 } & Hoja bandera & Madurez & & & \\
\hline FI50 \% + HMA & $78.1 \pm 1.8$ & $123.2 \pm 1.2 \mathrm{ab} *$ & $16.1 \pm 0.7 \mathrm{a}$ & $27.7 \pm 1.1 \mathrm{ab}$ & $6696 \pm 90 \mathrm{ab}$ \\
HMA & $76.4 \pm 2.2$ & $124.4 \pm 0.6 \mathrm{ab}$ & $15.7 \pm 1.2 \mathrm{a}$ & $28.8 \pm 0.7 \mathrm{a}$ & $7040 \pm 132 \mathrm{a}$ \\
Testigo & $75.6 \pm 2.0$ & $125.6 \pm 2.2 \mathrm{a}$ & $16.6 \pm 1.0 \mathrm{a}$ & $27.0 \pm 1.0 \mathrm{~b}$ & $6518 \pm 104 \mathrm{~b}$ \\
Significancia F & $74.6 \pm 2.3$ & $118.9 \pm 1.1 \mathrm{~b}$ & $13.7 \pm 0.5 \mathrm{~b}$ & $26.1 \pm 1.2 \mathrm{~b}$ & $6007 \pm 108 \mathrm{c}$ \\
\hline
\end{tabular}

*Letras distintas denotan diferencias significativas con la prueba de Tukey $(\mathrm{p}<0.05)$

FI100 \%: fertilización inorgánica $100 \%$ (120-40-00), FI50 \%: fertilización inorgánica $50 \%$ 
CUADRO IV. RESPUESTA DEL SORGO 83G19 (2016) EN ESTADO DE HOJA BANDERA Y MADUREZ FISIOLÓGICA CON DIFERENTE MANEJO DE FERTILIZACIÓN (FI) Y/O INOCULACIÓN DEL HMA

\begin{tabular}{lcccccc}
\hline Tratamiento & \multicolumn{2}{c}{ Altura de planta $(\mathrm{cm})$} & & $\begin{array}{c}\text { Diámetro de tallo } \\
(\mathrm{cm})\end{array}$ & $\begin{array}{c}\text { Longitud de panoja } \\
(\mathrm{cm})\end{array}$ & $\begin{array}{c}\text { Rendimiento } \\
(\mathrm{kg} / \mathrm{ha})\end{array}$ \\
\cline { 2 - 3 } & Hoja bandera & Madurez & & $12.7 \pm 0.7$ & $29.3 \pm 0.9 \mathrm{a}$ & $7616 \pm 376 \mathrm{a}$ \\
\hline 120-40-00 (FI100 \%) & $113.7 \pm 1.1$ & $146.8 \pm 1.5 \mathrm{a} *$ & & $12.9 \pm 1.0$ & $29.0 \pm 0.6 \mathrm{a}$ & $7312 \pm 407 \mathrm{a}$ \\
FI50 \% + HMA & $112.7 \pm 2.3$ & $146.4 \pm 1.3 \mathrm{a}$ & & $11.6 \pm 0.6$ & $28.0 \pm 0.8 \mathrm{a}$ & $6764 \pm 211 \mathrm{~b}$ \\
HMA & $111.3 \pm 3.7$ & $135.6 \pm 1.7 \mathrm{~b}$ & & $11.5 \pm 0.5$ & $26.6 \pm 0.5 \mathrm{~b}$ & $5987 \pm 410 \mathrm{c}$ \\
Testigo & $106.3 \pm 2.3$ & $133.1 \pm 1.8 \mathrm{~b}$ & & $11.5 \pm 0.01$ & 0.009 \\
Significancia F & 0.075 & 0.001 & 0.41 & 0.01 & \\
\hline
\end{tabular}

*Letras distintas denotan diferencias significativas con la prueba de Tukey $(\mathrm{p}<0.05)$

FI100 \%: fertilización inorgánica $100 \%$ (120-40-00); FI50 \%: fertilización inorgánica 50 \%

en el diámetro de tallo no se observaron diferencias significativas, a diferencia de lo sucedido con esta variable en 2011. Solamente en el estado de madurez fisiológica se observaron diferencias $(\mathrm{p}=0.001)$. La altura mayor de planta se registró con FI100 \% y FI50 \% más el HMA con promedio de $146.6 \mathrm{~cm}$, $12.2 \mathrm{~cm}$ de altura más que con que el HMA y el testigo, los cuales fueron semejantes con promedio de $134.3 \mathrm{~cm}$. La longitud de la panoja del testigo fue superada $(p=0.01)$ y con valores semejantes para los tres tratamientos con fertilización y/o inoculación micorrízica. El rendimiento de grano se incrementó significativamente con la fertilización convencional (FI100 \%) y con FI50 \% más HMA, en $22-27 \%$ respecto del el testigo. Además, el HMA superó en $13 \%$ el rendimiento del testigo (Cuadro IV).

Con relación a la biomasa seca foliar y radical, existieron variaciones significativas entre los tratamientos. La biomasa foliar del testigo fue superada $(\mathrm{p}=0.01)$ por el resto de los tratamientos. Una respuesta diferente se observó con la biomasa radical: los tratamientos que llevaron la inoculación del HMA promovieron el mayor peso radical $(\mathrm{p}=$ 0.007), comparado con la fertilización convencional y el testigo (Fig. 1).

Los coeficientes de correlación indicaron que el nivel de SPAD y la longitud de panoja fueron las variables que estuvieron más estrechamente relacionadas con el rendimiento de grano (Cuadro V). Esto se puede explicar por el hecho de que el nivel de clorofila refleja el estatus nutricional de la planta y la longitud de panoja es un componente de rendimiento.

En general, los mayores rendimientos obtenidos en cada año y en el promedio de ambos años, fueron los que propiciaron la fertilización convencional y el $50 \%$ del fertilizante más el HMA. Sin embargo, el análisis económico de 2011 indicó que se obtuvo menor rentabilidad (b/c) con la fertilización convencional, inclusive superada por el testigo; en 2016,

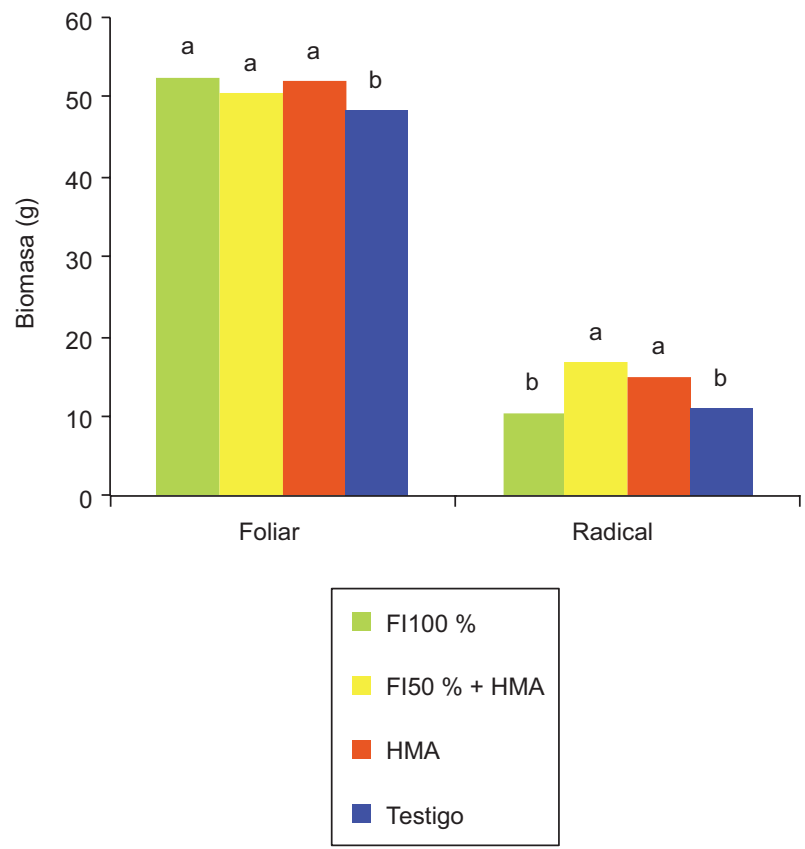

Fig. 1. Biomasa foliar y radical seca en el sorgo $83 \mathrm{~g} 19$ obtenida con fertilización inorgánica (fi) y/o micorrización (HMA). Letras distintas denotan diferencias significativas con la prueba de Tukey $(\mathrm{p}<0.05)$

los tratamientos con el HMA solo o en combinación con FI50 \%, fueron los más rentables, aunque en el promedio de los dos años la inoculación del HMA sola registró la mayor rentabilidad con $1.50 \mathrm{~b} / \mathrm{c}$ (Cuadro VI).

\section{DISCUSIÓN}

Hasta el estado de hoja bandera del sorgo DKS60 (2011), no se observaron variaciones significativas en el SPAD por efecto de los tratamientos. Por el contrario, la respuesta de 83G19 (2016) mostró diferencias 
CUADRO V. COEFICIENTES DE DETERMINACIÓN ENTRE CARACTERÍSTICAS DE PLANTA Y RENDIMIENTO DE GRANO DE SORGO $(n=7)$

\begin{tabular}{lll}
\hline Variable & Ecuación & $\mathrm{R}^{2}$ \\
\hline Clorofila SPAD y rendimiento & $\mathrm{y}=0.006 \mathrm{x}+3.463$ & 0.94 \\
Altura de planta en hoja bandera y rendimiento & $\mathrm{y}=0.0077 \mathrm{x}+145.2$ & 0.25 \\
Altura de planta en madurez y rendimiento & $\mathrm{y}=0.0127 \mathrm{x}+45.91$ & 0.47 \\
Diámetro de tallo y rendimiento & $\mathrm{y}=0.0019 \mathrm{x}+0.667$ & 0.32 \\
Longitud de panoja y rendimiento & $\mathrm{y}=453.01 \mathrm{x}-6043.6$ & 0.78 \\
\hline
\end{tabular}

CUADRO VI. RENDIMIENTO DE GRANO DE SORGO (kg/ha) Y RELACIÓN BENEFICIO-COSTO (B/C) ASOCIADOS A LOS TRATAMIENTOS EN 2011 Y 2016. CERIB

\begin{tabular}{lcccccc}
\hline Tratamiento & 2011/DKS60 & B/C & $2016 / 83 \mathrm{G} 19$ & $\mathrm{~B} / \mathrm{C}$ & Promedio & $\mathrm{B} / \mathrm{C}$ \\
\hline 120-40-00 (FI100 \%) & 6696 & 1.2 & 7616 & 1.4 & 7156 & 1.30 \\
FI50 \% + HMA & 7040 & 1.4 & 7312 & 1.5 & 7176 & 1.45 \\
HMA & 6518 & 1.5 & 6764 & 1.5 & 6641 & 1.50 \\
Testigo & 6007 & 1.4 & 5987 & 1.3 & 5997 & 1.35 \\
\hline
\end{tabular}

FI100 \%: fertilización inorgánica 100 \% (120-40-00), FI50 \%: fertilización inorgánica 50 \%

en los valores SPAD, que fueron ascendentes desde el estado de cinco hojas hasta la floración, los cuales fueron superiores al testigo con los tratamientos fertilizados y/o inoculados con HMA ( $R$. intraradices). La diferente respuesta SPAD de los híbridos en el estado de hoja bandera, podría atribuirse al genotipo, más que a las condiciones edáficas, ya que con excepción de la textura el nivel de fertilidad fue similar en ambos años (Cuadro I). Las lecturas SPAD se hicieron en las hojas bandera, ya que éstas desempeñan un papel primordial como fuente de fotosintatos durante el desarrollo del grano, y la ausencia de nitrógeno afecta la actividad fotosintética. Es posible que el incremento ascendente de SPAD en el híbrido 83G19 en particular (Cuadro II) se deba a la absorción progresiva de nitrógeno y su movilidad a hojas jóvenes a través del ciclo del cultivo. Un patrón similar fue reportado por Díaz y Garza (2006), quienes inocularon con HMA genotipos de pasto buffel (Cenchrus ciliaris). En especies distintas a gramíneas, plantas de trébol blanco (Trifolium repens) inoculadas con Diversispora versiformis y Paraglomus occultum registraron las mayores concentraciones de clorofilas $\mathrm{a}, \mathrm{b}$ y a + b comparadas con plantas testigo ( $\mathrm{Lu} \mathrm{y} \mathrm{Wu}$ 2017). Cardarelli et al. (2010) registraron en calabacita (Cucurbita pepo) inoculada con $R$. intraradices los mayores SPAD, relacionados con un mejor estatus nutricional en nque destacaron N, P, K, Fe, Zn y Mn en el follaje.
La altura de planta en el estado de hoja bandera no mostró diferencias entre los tratamientos en ambos experimentos. Lo anterior podría deberse a que las necesidades nutrimentales de la planta en ese estado de desarrollo fueron solventadas por el nivel de fertilidad inicial del suelo. Esta respuesta se ha registrado también en otras especies con inoculación micorrízica. Desgan et al. (2008) informaron que en plantas de tomate (Lycopersicon esculentum), la altura y el número de hojas de la planta fueron semejantes hasta los 40 días después del trasplante, con o sin micorrización. De igual forma, Castillo et al. (2009) registraron alturas de planta de chile (Capsicum annuum) similares hasta los 60 días después de la siembra con o sin inoculación micorrízica; posteriormente, la mayor altura de planta se alcanzó con HMA. Por el contrario, estudios en maíz (Zea mays) demostraron diferente altura de planta y biomasa con fertilización inorgánica durante los primeros estadios del desarrollo (El-Handi y Woodard 2008).

En cuanto a la madurez fisiológica, tanto en 2011 como en 2016 los tratamientos afectaron significativamente las características de planta evaluadas y el rendimiento del grano, con excepción del diámetro de tallo en 2016. El diámetro de tallo de sorgo tiene particular importancia para evitar el acame de las plantas. También en 2016 se destacó la mayor promoción de biomasa foliar con los tratamientos fertilizados y/o inoculados, los cuales superaron al testigo, aunque 
fue evidente que los mayores valores de biomasa radical se observaron con los tratamientos que incluyeron inoculación micorrízica. Esta peculiaridad favorece una mayor disponibilidad de nutrientes dada la expansión o volumen del sistema radical. Diversos autores han señalado el beneficio del incremento de la biomasa radical en plantas inoculadas con HMA (Castillo et al. 2009, Díaz et al. 2013, Lu y Wu 2017). La contribución de los HMA al aumento de la biomasa vegetal se encuentra ligada a mecanismos que conllevan la producción de fitohormonas, así como al crecimiento radical, que aumenta el volumen de suelo explorado, con un consecuente incremento en la captación de nutrientes y agua (Smith y Read 2008, Yang et al. 2014).

En general, en 2016 el 83G19 registró las mejores características de planta en hoja bandera y en madurez fisiológica. Esto puede obedecer a múltiples factores tanto inherentes al genotipo como al año, ya que la temperatura y precipitación promedio fueron de 27.5 y $25.1^{\circ} \mathrm{C}$, y 95 y $121 \mathrm{~mm}$ en 2011 y 2016 , respectivamente. Todos los tratamientos superaron al testigo en las características de planta y rendimiento de grano. Lo anterior muestra lo que está documentado localmente: el sorgo responde tanto a fertilización inorgánica (Salinas 2008) como la micorrización con $R$. intraradices (Díaz et al. 2013).

Los resultados obtenidos en el estudio demuestran el impacto de la micorrización sola o con la reducción del fertilizante mineral sobre la promoción vegetal y el rendimiento de sorgo. Está documentado que los HMA tienen un efecto simbiótico como facilitadores en la absorción de nutrimentos para la planta. Thirkell et al. (2016) y Scandellari (2017) demostraron a través de ${ }^{15} \mathrm{~N}$ que los HMA incrementan la adquisición de $\mathrm{N}$ por las plantas mediante las hifas externas. Los HMA pueden secretar fosfatasas para hidrolizar el $\mathrm{P}$ de compuestos orgánicos, lo cual incrementa la productividad de los cultivos en condiciones deficientes de P (Rouphael et al. 2015). Miller (2000) señaló que el micelio de los hongos micorrízicos transporta el $\mathrm{P}$ del suelo a la planta inmediatamente después de que entra en conexión con el sistema radical en desarrollo. Las hifas extraradicales también facilitan la absorción de micronutrientes inmóviles como $\mathrm{Cu}, \mathrm{Zn}$, y otros cationes como K, Ca, Mg y Fe (Smith y Read 2008, Malusá et al. 2016). Además de mejorar la nutrición de las plantas, los HMA interfieren con el balance hormonal, ya que influyen como bioreguladores en el desarrollo de las plantas y mitigan los efectos de estrés ambiental (Rouphael et al. 2015); esto no sólo permite el incremento de la biomasa y el rendimiento de los cultivos, sino que también puede favorecer cambios en parámetros de calidad (Antunes et al. 2012, Bona et al. 2015). Al respecto, se demostró que la capacidad simbiótica de la micorrización en sorgo, indujo un contenido similar de proteína en el grano respecto a la fertilización con $80 \mathrm{~kg} / \mathrm{ha}$ de $\mathrm{N}$ (Díaz et al. 2008).

Los efectos que tuvieron la complementariedad de la reducción de la fertilización inorgánica con el HMA, así como el HMA independiente, tienen relevancia en el ambiente. Ambos manejos se pueden considerar como estrategias agronómicas que permiten mejorar la eficiencia de los cultivos e inducir menor impacto negativo en el entorno agroecológico (Adesemoye y Kloepper 2009, Malusá et al. 2016). Es necesario conocer los efectos de la asociación entre cepas de HMA eficientes y niveles de fertilizantes minerales en los cultivos, de acuerdo con las condiciones particulares de los agrosistemas (Berruti et al. 2015, Ziane et al. 2017). Por ejemplo, el máximo rendimiento de trigo (Triticum aestivum) se registró con la combinación de $R$. intraradices y el $75 \%$ de N-P-K adicionado al suelo (Sharma et al. 2012). De manera similar, Díaz et al. (2015) informaron que tanto la mayor longitud de la panoja como el rendimiento de grano de sorgo se registraron con la combinación de bioinoculantes más el $50 \%$ del fertilizante y la fertilización convencional (100\%). De acuerdo con Bona et al. (2015), la fresa (Fragaria spp.) con inoculante comercial a base de $R$. intraradices, G. ageratum, G. viscosum, Claroideoglomus etunicatum y C. claroideum más el $70 \%$ del fertilizante, obtuvo mayor número y tamaño de frutos y rendimiento, comparado con plantas no inoculadas y con fertilización convencional.

Con relación al indicador de rentabilidad de la producción, se demostró que los tratamientos FI50 \% + HMA y HMA obtuvieron b/c de 1.45 y 1.5 , respectivamente, superiores a los obtenidos con fertilización convencional. Lo anterior, pese a que la inoculación con HMA registró un rendimiento de $852 \mathrm{~kg} / \mathrm{ha}$ menor que la fertilización completa. Estos resultados coinciden con los obtenidos por Salinas (2008), quien comparó durante cuatro años consecutivos el manejo con fertilización convencional contra micorrización con $R$. intraradices en sorgo de riego. El promedio de rendimiento con la primera fue de $5.3 \mathrm{t} / \mathrm{ha}$ y de 4.8 t/ha para la segunda; no obstante, la micorrización alcanzó un b/c de 3.0 contra 2.5 con fertilización tradicional. En la producción comercial de sorgo de temporal, es común el empleo de micorrización sin adición de fertilizantes durante años (Díaz et al. 2014), así como en el manejo de sorgo orgánico. Además de la incorporación de los residuos de co- 
secha, la incorporación de abonos orgánicos podría representar un manejo alternativo.

Es importante comprender mejor las complejas interacciones entre los hongos micorrízicos, la fertilización mineral y la planta, con el propósito de reducir o sustituir las cantidades de fertilizantes aplicados, reducir los costos sin afectar la producción, y disminuir tanto el agotamiento de la fertilidad con el tiempo como los efectos nocivos al ambiente. No obstante, la información sobre la funcionalidad de los HMA que influye directa e indirectamente, parcial o totalmente en las modificaciones de las propiedades físicas, químicas y biológicas del suelo a través del tiempo, es limitada, ya que las variaciones dependen de las características del suelo y de su capacidad amortiguadora nutrimental en la interacción suelo-planta-HMA (Mader et al. 2004, Yang et al. 2014). Díaz et al. (2014) compararon sorgo micorrizado vs. fertilizado después de cinco años, registrando similares características de plantas en el último año; sin embargo, en las inoculadas con mayor desarrollo radical, el rendimiento fue semejante aunque $17.7 \%$ más rentable con micorrización. También las propiedades físicas y químicas del suelo fueron semejantes, aunque la micorrización incrementó el número de esporas de HMA y la biomasa microbiana. Liere et al. (2017) señalaron que el uso excesivo de agroquímicos ha tenido como resultado contaminación, decremento de la biodiversidad en las regiones agrícolas, degradación de los agroecosistemas e incrementos en los costos de producción. Las prácticas implementadas para la producción agrícola sostenible podrían reemplazar a la agricultura tradicional, aunque para eso se requiere de una mayor comprensión y entendimiento de las interacciones bilógicas dentro de los agroecosistemas.

En el presente estudio se demostraron las similitudes en las características de planta y el rendimiento del grano de sorgo entre la fertilización inorgánica convencional y la combinación del HMA $R$. intraradices con el $50 \%$ de la fertilización, así como la rentabilidad potencial de la producción que ofreció la inoculación sola de $R$. intraradices. La inoculación con el HMA promovió una producción de sorgo eficiente y rentable, debido al menor costo por insumos, así como la reducción de riesgos por contaminación ambiental. Xiang et al. (2012) han destacado que los biofertilizantes, además del efecto benéfico en la productividad agrícola, tienen gran relevancia en los agroecosistemas debido a que pueden reducir o sustituir el uso de la fertilización mineral y su consecuente contaminación del entorno. Calvo et al. (2013) enfatizaron que para reducir las emisiones de $\mathrm{N}_{2} \mathrm{O}$ asociadas con la aplicación de fertilizantes nitrogenados, es importante considerar el uso de bioinoculantes como parte del manejo agronómico. Desde el punto de vista económico y ecológico, la reducción de la fertilización inorgánica a través de bioinoculantes puede representar una práctica viable que promueva mayor rentabilidad, así como la conservación agroecológica de los sistemas de producción y la biodiversidad.

\section{CONCLUSIONES}

Los valores de clorofila, altura de planta, diámetro de tallo, longitud de panoja y rendimiento de grano fueron diferentes entre los tratamientos. En general, los mayores valores se registraron con la fertilización convencional (120-40-00) y la mitad de ésta más la inoculación de $R$. intraradices, en tanto que la biomasa radical fue superior con los dos tratamientos que incluyeron el HMA. Con estos mismos tratamientos se obtuvo la mayor rentabilidad de la producción de sorgo $(1.45-1.5 \mathrm{~b} / \mathrm{c})$.

\section{AGRADECIMIENTOS}

Se agradece el apoyo del Patronato para la Investigación, Fomento y Sanidad Vegetal, a través del proyecto No. 18365633858 "Demandas de Investigación, Validación y Transferencia de Tecnología de Productores Agrícolas Organizados del Norte de Tamaulipas". Asimismo, al Sr. Juan Olvera Martínez por la asistencia en las labores de campo.

\section{REFERENCIAS}

Adesemoye A.O. y Kloepper J.W. (2009). Plant-microbes interactions in enhanced fertilizer-use efficiency. Appl. Microbiol. Biotechnol. 85 (1), 1-12.

DOI: $10.1007 / \mathrm{s} 00253-009-2196-0$

Allen B.L., Jolley V.D., Robbins C.W. y Freeborne L.L. (2001). Follow wheat cropping of unamended and manure-amended soil related to mycorrhizal colonization, yield and plant nutrition of dry bean and sweet corn. J. Plant Nutr. 24 (3), 921-943.

DOI: $10.1081 /$ pln-100103782

Antunes P., Franken P., Schwarz D., Rillig M., Cosme M., Scott M. y Hart M. (2012). Linking soil biodiversity and human health: do arbuscular mycorrhizal fungi contribute to food nutrition. En: Soil ecology 
and ecosystem services (Wall D.H., Bardgett R.D, Behan-Pelletier V., Herrick H., Jones J.E., Ritz K., Six J., Strong D.R. y van der Putten W.H., Eds.). Oxford University Press, Nueva York, NY, EUA, pp. 153-172.

Berruti A., Lumini E., Balestrini R. y Bianciotto G. (2015). Arbuscular mycorrhizal fungi as natural biofertilizers: Let's benefit from past successes. Front Microbiol. 6, 1559. DOI: $10.3389 /$ fmicb. 2015.01559

Bona E., Lingua G., Manassero P., Cantamessa S., Marsano F., Todeschini V., Copetta A., D’Agostino G., Massa N., Avidano L., Gamalero E. y Berta G. (2015). AM fungi and PGP Pseudomonas increase flowering, fruit production, and vitamin content in strawberry grown at low nitrogen and phosphorus levels. Mycorrhiza 25 (3), 181-193. DOI: 10.1007/s00572-014-0599-y

Calvo P., Watts D.B., Ames R.N., Kloepper J.W. y Torbert H.A. (2013). Microbial-based inoculants impact nitrous oxide emissions from an incubated soil medium containing urea fertilizers. J. Environ. Qual. 42 (3), 704-712. DOI: $10.2134 /$ jeq2012.0300

Cardarelli M., Rouphael Y., Rea E. y Colla G. (2010). Mitigation of alkaline stress by arbuscular mycorrhiza in zucchini plants grown under mineral and organic fertilization. J. Plant Nutr. Soil Sci. 173 (5), 778-787. DOI: $10.1002 /$ jpln.200900378

Castillo R.C., Sotomayor S.L., Ortiz O.C., Leonelli C.G., Borie F.B. y Rubio H.R. (2009). Effect of arbuscular mycorrhizal fungi on an ecological crop of chili peppers (Capsicum annuum L.) Chilean J. Agr. Res. 69 (1), 79-87.

Desgan Y.H., Kusvaran S. y Ortas I. (2008). Responses of soilless grown tomato plants to arbuscular mycorrhizal fungal (Glomus fasciculatum) colonization in recycling and open systems. Afr. J. Biotech. 7, 3606-3613.

Díaz F.A. y Garza C.I. (2006). Colonización micorrízica arbuscular y crecimiento de genotipos de pasto buffel (Cenchrus ciliaris). Rev. Fitotec. Mex. 29 (3), 203-206.

Díaz F.A., Garza C.I., Pecina Q.V. y Montes G.N. (2008). Respuesta del sorgo a micorriza arbuscular y Azospirillum en estrés hídrico. Rev. Fitotec. Mex. 31 (2), 35-42.

Díaz F.A., Cortinas E.H., Valadez G.J., De la Garza C.M. y Peña del Río M.A. (2013). Micorriza arbuscular en sorgo bajo diferente manejo agrotecnológico y ambiental. Rev. Mex. Cien. Agr. 4 (2), 215-228.

Díaz F.A., Salinas G.J., Espinosa S.F., Peña M.A., Garza R.F. y Grageda C.O. (2014). Características de planta, suelo y productividad entre sorgo fertilizado e inoculado con micorriza arbuscular. Rev. Mex. Cien. Agr. 3 (3), 379-390.

Díaz F.A., Gálvez L.D. y Ortiz C.F. (2015). Bioinoculación y fertilización química reducida asociadas con el crecimiento de planta y productividad de sorgo. Rev. Int. Contam. Ambie. 31 (3), 245-252.
Díaz M.R., Díaz F.A., Garza C.I. y Ramírez L.A. (2007). Brassinoesteroides e inoculación de micorriza arbuscular (Glomus intraradices) en el crecimiento y producción de sorgo en campo. Terra Latinoamer. 25 (3), 77-83.

El-Handi K.H. y Woodard H.J. (2008). Response of early corn growth to fertilizer rates and placement methods. J. Plant Nutr. 18, 1103-1120.

DOI: 10.1080101904169509364966

FIRA (2016). Panorama agroalimentartio: sorgo 2016. Fideicomiso Instituido en Relación con la Agricultura. Dirección de Investigación y Evaluación Económica y Sectorial. Ciudad de México, 34 pp.

Grageda C.O., Díaz F.A., Peña C.J. y Vera N.J. (2012). Impacto de los biofertilizantes en la agricultura. Rev. Mex. Cien. Agr. 3 (6), 1261-1274.

Liere H., Jha S. y Philipott S.M. (2017). Intersection between biodiversity conservation, agroecology, and ecosystem services. Agroecol. Sustain. Food Systems 41 (3), 723-760. DOI: $10.1080 / 21683565.2017 .1330796$

Lu L. y Wu O. (2017). Mycorrhizas promote plant growth, root morphology and chlorophyll production in white clover. Biotechnol. 16 (1), 34-39.

DOI: 10.3923/biotech.2017.34.39

Mader P., Endenhofer S., Boller T., Wienken A. y Niggli U. (2004). Arbuscular mycorrhizae in long-term field trail comparing low-input (organic, biological) and high-input (conventional) farming systems in a crop rotation. Biol. Fert. Soils 31 (2), 150-156.

DOI: $10.1007 / \mathrm{s} 003740050638$

Malusá E. Pinzari F. y Canfora L. (2016). Efficacy of biofertilizers: Challenges to improve crop production. En: Microbial inoculants in sustainable agricultural productivity (Singh D.P., Singh H.B. y Ratna P., Eds.). Springer, Nueva Delhi, India, pp. 373-392.

DOI: 10.1007/978-81-322-2644-4_2

Miller M.H. (2000). Arbuscular mycorrhizae and the phosphorus nutrition of maize: A review of Guelph studies. Can. J. Plant Sci. 80 (1), 47-52.

DOI: $10.4141 / \mathrm{p} 98-130$

Rosales R.E., Montes G.N. y Reyes M.C. (2006). Tecnología para la producción de sorgo en el norte de Tamaulipas. Memoria Técnica No. 2. Campo Experimental Río Bravo, Instituto Nacional de Investigaciones Forestales, Agrícolas y Pecuarias, Río Bravo, Tamaulipas, México, pp. 61.

Rose M.T., Phuong T.L., Nhan D.K., Cong P.T., Yhanh H.N. y Kennedy I.R. (2014). Up to $52 \% \mathrm{~N}$ fertilizer replaced by biofertilizer in lowland rice via farmer participatory research. Agr. Sustainable Develop. 34 (4), 857-868.

DOI: $10.1007 / \mathrm{s} 13593-014-0210-0$ 
Rouphael Y., Franken P., Schneider C., Schwarz D., Giovannetti M., Agnolucei M., Pascale S., Bonini P. y Colla G. (2015). Arbuscular mycorrhizal fungi act as biostimulants in horticultural crops. Sci. Hort. 196, 91-108.

DOI: $10.1016 /$ j.scienta.2015.09.002

Salinas G.J. (2008). Eficiencia en la fertilización en los cultivos de maíz y sorgo. Campo Experimental Río Bravo, Instituto Nacional de Investigaciones Forestales, Agrícolas y Pecuarias. Publicación Especial No. 34. Río Bravo, Tamaulipas, México, 28 pp.

Scandellari F. (2017). Arbuscular mycorrhizal contribution to nitrogen uptake of grapevines. Vitis J. Grap. Res. 56 (4), 147-154.

DOI: $10.5073 /$ vitis.2017.56.147-154

SEMARNAT (2002). Norma Oficial Mexicana NOM021-SEMARNAT. Que establece las especificaciones de fertilidad, salinidad y clasificación de suelos, estudios, muestreo y análisis. Secretaría del Medio Ambiente y Recursos Naturales. Diario Oficial de la Federación, 31 de diciembre.

Sharma S., Gupta R., Dugar G. y Srivastava A. (2012). Impact of application of biofertilizers on soil structure and resident microbial community structure and function. En: Bacteria in agrobiology: Plant probiotics (Maheshwari D.K., Ed.). Hauz Khas, Nueva Delhi, India, pp. 65-79.

Silva S.M., Medina G.G., Ruiz C.J., Díaz P.G. y Cano G.M. (2007). Estadísticas climatológicas del Estado de Tamaulipas. Libro Técnico No. 2. Campo Experimental Río Bravo, Instituto Nacional de Investigaciones Forestales, Agrícolas y Pecuarias. Río Bravo, Tamaulipas, México, 316 pp.

Smith S.E. y Read D.J. (2008). Mycorrhizal symbiosis. $3 a$. ed. Academic Press, Londres, Reino Unido, 320 pp.
Sylvia D.M., Hammond L.C., Bennett J.M., Hass J.H., y Linda S.B. (1993). Field response of maize to a VAM fungus and water management. Agron. J. 85 (4), 193-198.

DOI: 10.2134/agronj1993.00021962008500020006x

Thirkell T.J., Cameron D.D. y Hodge A. (2016). Resolving the 'nitrogen paradox' of arbuscular mycorrhizas: fertilization with organic matter brings considerable benefits for plant nutrition and growth. Plant Cell Environ. 39 (8), 1683-1690.

DOI: $10.1111 /$ pce. 12667

Williams A.H., Montes G.N. y Pecina Q.V. (2006). Sorgo. En: Campo Experimental Río Bravo: 50 años de investigación agropecuaria en el norte de Tamaulipas (Rodríguez del Bosque L., Ed.). Libro Técnico No. 1. Campo Experimental Río Bravo, Instituto Nacional de Investigaciones Forestales, Agrícolas y Pecuarias. Río Bravo, Tamaulipas, México, 287 pp.

Xiang W., Zhao L., Xu X., Qin Y. y Yu G. (2012). Mutual information flow between beneficial microorganisms and the roots of host plants determined the biofunctions of biofertilizers. Amer. J. Plant Sci. 3 (4), 1115-1120. DOI: 10.4236/ajps.2012.38134

Yang C., Ellouze W., Borrell A., Taheri A., Dai M., Kabir Z. y Hamel C. (2014). Management of the arbuscular mycorrhizal symbiosis in sustainable crop production. En: Mycorrhizal fungi: Use in sustainable agriculture and land restoration (Solaiman Z.M., Ed.). SpringerVerlag, Berlín, Heidelberg, Alemania, pp. 89-118. DOI: 10.1007/978-3-662-45370-4_7

Ziane H., Hamza A.M., Beddiar A. y Gianinazzi S. (2017). Effects of arbuscular mycorrhizal fungi and fertilization levels on industrial tomato growth and production. Int. J. Agr. Biol. 19 (1), 341-347.

DOI: $10.17957 / \mathrm{ijab} / 15.0287$ 